TITLE:

\title{
Exciton localization of single-walled carbon nanotubes revealed by femtosecond excitation correlation spectroscopy
}

\section{$\operatorname{AUTHOR}(\mathrm{S}):$}

Hirori, Hideki; Matsuda, Kazunari; Miyauchi, Yuhei; Maruyama, Shigeo; Kanemitsu, Yoshihiko

\section{CITATION:}

Hirori, Hideki ...[et al]. Exciton localization of single-walled carbon nanotubes revealed by femtosecond excitation correlation spectroscopy. Physical Review Letters 2006, 97(25): 257401.

ISSUE DATE:

2006-12

URL:

http://hdl.handle.net/2433/87359

RIGHT:

c 2006 The American Physical Society 


\title{
Exciton Localization of Single-Walled Carbon Nanotubes Revealed by Femtosecond Excitation Correlation Spectroscopy
}

\author{
Hideki Hirori, ${ }^{1}$ Kazunari Matsuda, ${ }^{1, *}$ Yuhei Miyauchi, ${ }^{2}$ Shigeo Maruyama, ${ }^{2}$ and Yoshihiko Kanemitsu ${ }^{1, \dagger}$ \\ ${ }^{1}$ Institute for Chemical Research, Kyoto University, Gokasho, Uji, Kyoto 611-0011, Japan \\ ${ }^{2}$ Department of Mechanical Engineering, The University of Tokyo, 7-3-1 Hongo, Bunkyo-ku, Tokyo 113-8656, Japan
}

(Received 3 September 2006; published 18 December 2006)

\begin{abstract}
Photoluminescence (PL) dynamics in single-walled carbon nanotubes (SWNTs) has been studied by the femtosecond excitation correlation method with a $150 \mathrm{fs}$ time resolution. The SWNT samples were synthesized by different methods and suspended in gelatin films or $\mathrm{D}_{2} \mathrm{O}$ solutions. The PL dynamics of SWNTs depends on the local environment surrounding the SWNTs rather than the synthesis methods. The very weak temperature dependence of $\tau_{\mathrm{PL}}$ and the environment-dependent $\tau_{\mathrm{PL}}$ reveal that the PL relaxation process is dominated by the interplay between free excitons and weakly localized excitons.
\end{abstract}

Electronic and optical properties of single-walled carbon nanotubes (SWNTs) have attracted considerable attention in terms of both fundamental physics and technological applications to optoelectronic devices [1,2]. The discovery of efficient photoluminescence (PL) from isolated semiconducting SWNTs has allowed us to study the various optical properties of SWNTs in detail [3]. Recently, both theoretical predictions and experimental studies have shown that the optical properties of SWNTs are determined by the dynamics of excitons formed by the strong electronhole interaction in the quasi-1D systems [4-13].

Time-resolved PL spectroscopy has been one of the most powerful techniques to elucidate the microscopic mechanism of exciton dynamics in SWNTs. Several researchers reported the PL lifetimes of SWNTs, and their values range from 10 to $180 \mathrm{ps}$ [14-19]. These values of PL lifetimes observed experimentally are much shorter than the radiative lifetimes calculated from the quantum efficiency of nanotubes $(\sim 110 \mathrm{~ns})$ [14] and by the theoretical prediction $(\sim 10 \mathrm{~ns})[20,21]$. These results imply that the observed PL lifetimes are determined by the nonradiative recombination process. The broad distribution of the observed PL lifetimes may be due to the differences in samples, the local environment of the SWNTs, the number of impurities or defects, and so on. In addition, the time resolution of most recent PL decay measurements for SWNTs is greater than $\sim 10 \mathrm{ps}$ and is comparable to the measured nonradiative decay lifetime [15-19]. From these standpoints, in order to clarify the mechanism of exciton dynamics in SWNTs, it is necessary to measure systematically the PL decay of different SWNT samples with higher time resolutions.

In this Letter, we have studied PL lifetimes $\left(\tau_{\mathrm{PL}}\right)$ for four different SWNT samples by means of the femtosecond excitation correlation (FEC) method with a 150 fs time resolution. The SWNTs were synthesized by the alcohol catalytic chemical vapor-deposition (ACCVD) method and the high-pressure $\mathrm{CO}$ (HiPco) method and were suspended in gelatin films or $\mathrm{D}_{2} \mathrm{O}$ solutions. The results showed that the PL lifetime of SWNTs in $\mathrm{D}_{2} \mathrm{O}$ solutions $\left(\tau_{\mathrm{PL}} \sim 22 \mathrm{ps}\right)$ is faster than that in gelatin films $\left(\tau_{\mathrm{PL}} \sim 31 \mathrm{ps}\right)$, whereas the PL lifetimes scarcely depend on the synthesis method and the tube diameters of SWNTs. The temperature dependence of $\tau_{\mathrm{PL}}$ for the SWNTs in gelatin films reveals that the relaxation processes of excitons are determined by the interplay of free excitons and weakly localized excitons in potential fluctuations due to changes in the local environment rather than the intrinsic effects such as the exchange splitting of excitons.

Four kinds of samples were prepared as follows: The SWNTs were synthesized by the ACCVD method at $750{ }^{\circ} \mathrm{C}$ [22] and by the HiPco method [23] (batch no. HPR 113.3 from Rice University). Both synthesized SWNTs were isolated by dispersion in $\mathrm{D}_{2} \mathrm{O}$ solutions with $0.5 \mathrm{wt} \%$ sodium dodecyl sulfate (SDS), vigorous sonication, and centrifugation at an acceleration of $330000 \mathrm{~g}$ for $1 \mathrm{~h}$ according to the procedure developed by O'Connell et al. [3]. The SWNTs in $\mathrm{D}_{2} \mathrm{O}$ solutions were used as the ACCVD- $\mathrm{D}_{2} \mathrm{O}$ and HiPco- $\mathrm{D}_{2} \mathrm{O}$ samples [24]. The SWNTs in gelatin films (the ACCVD-gelatin and HiPco-gelatin samples) were prepared by mixing the sodium dodecylbenzene sulfonate (SDBS)-suspended SWNTs in $\mathrm{D}_{2} \mathrm{O}$ solutions with the granulated powder of gelatin $(9 \mathrm{wt} \%)$, casting the solution on $\mathrm{SiO}_{2}$ substrates, and drying it at room temperature.

In the FEC experiments, the samples are excited with optical pulses from a Ti:sapphire laser of central wavelength $730 \mathrm{~nm}$, repetition rate $80 \mathrm{MHz}$, pulse duration 150 fs (full-width-at-half-maximum intensity - FWHM), and spectral width $8 \mathrm{~nm}$ (FWHM). The FEC method has been successfully applied to a variety of materials to measure the recombination lifetimes of free and bound excitons [25], because the time resolution of this method is limited only by the laser pulse duration. The two beams separated by the delay time $\tau$ were, respectively, chopped at 1000 and $800 \mathrm{~Hz}$ and collinearly focused onto the same spot $(\sim 10 \mu \mathrm{m})$ at $0^{\circ}$ incidence. Only the PL signal components modulated at the sum frequency $(1800 \mathrm{~Hz})$ were detected 
as FEC signals with a photomultiplier tube and a lock-in amplifier after dispersion of PL by a monochromator.

Shown in Fig. 1 are the time-integrated PL spectra of the SWNT samples under continuous-wave $\mathrm{He}-\mathrm{Ne}$ laser $(1.96 \mathrm{eV})$ excitation at room temperature [26]. The PL is associated with radiative emission from the lowest excited state in SWNTs, and a chiral index $(n, m)$ of each PL peak is assigned according to the data reported by Bachilo et al. $[27,28]$. As shown in Fig. 1, the PL spectral shape of the HiPco- $\mathrm{D}_{2} \mathrm{O}$ sample is slightly different from that of the ACCVD- $\mathrm{D}_{2} \mathrm{O}$ sample, because the HiPco sample consists of nanotubes with a broader distribution of chiral indices [29]. It is noteworthy that the thermogravimetric analysis and transmission electron microscopy images show that the HiPco samples include more metallic impurities and/or structural defects than the ACCVD ones [22,23,29]. In Fig. 1, the addition of gelatin causes small redshifts in the peak energies and a slight broadening of the linewidth compared to the SDS-suspended SWNT solutions. These behaviors may be caused by the transition energy shifts due to strains, the dielectric screening of the Coulomb interaction, structural defects along a nanotube, and their spatial nonuniformity [30,31]. In the inset in Fig. 1, the PL intensity of $(9,4)$ nanotubes in the ACCVD- $\mathrm{D}_{2} \mathrm{O}$ sample is shown to increase nonlinearly with increasing intensity of the optical pulses under $1.70 \mathrm{eV}$ excitation. Similar nonlinearities are observed for the other samples and nanotube species with different diameters, and they are caused by the phase-space filling of exciton states [32]. These nonlinearities allow us to obtain FEC signals as a function of delay time between two optical pulses.

Figure 2(a) shows the time evolution of FEC signals of $(9,4)$ nanotubes in the ACCVD- $\mathrm{D}_{2} \mathrm{O}$ sample under $1.70 \mathrm{eV}$

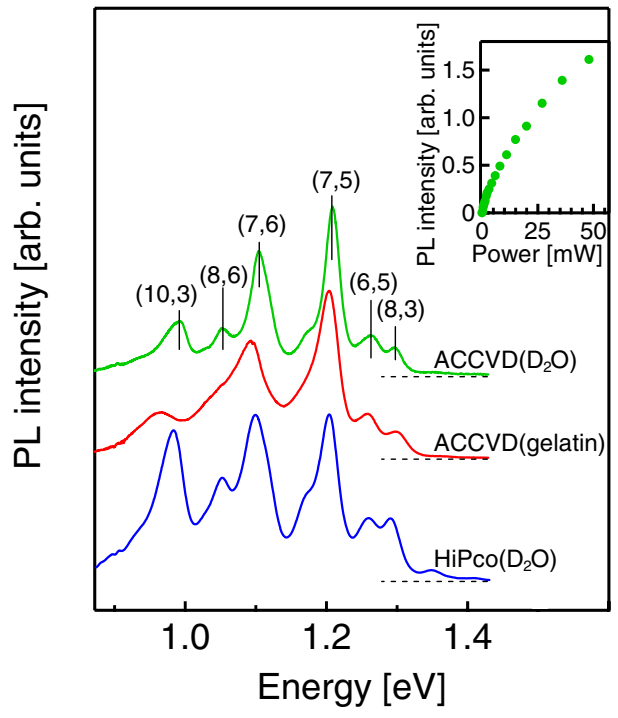

FIG. 1 (color online). PL spectra of the ACCVD- $\mathrm{D}_{2} \mathrm{O}$, ACCVD-gelatin, and HiPco- $\mathrm{D}_{2} \mathrm{O}$ samples under $1.96 \mathrm{eV}$ excitation. The inset shows the intensity dependence of PL amplitude for $(9,4)$ nanotubes in the ACCVD- $\mathrm{D}_{2} \mathrm{O}$ samples with the optical pulses under $1.70 \mathrm{eV}$ excitation. excitation at $5 \mathrm{~mW}$. The upward direction on the vertical axis indicates a negative sign of the FEC signals. The measured temporal profiles consist of fast decay $(<50 \mathrm{ps})$ and long tail ( $\gg 200 \mathrm{ps}$ ), which is consistent with previous observation [16]. However, since the slow decay time is comparable with the pulse repetition time in our measurements, we fit the PL decay with a single exponential function $A \exp \left(-\tau / \tau_{\mathrm{PL}}\right)+B$, where $A$ and $B$ are constants and $\tau_{\mathrm{PL}}$ is the lifetime of the fast-decay PL component as shown in Fig. 2(a). We find that the fitted curve well describes the experimentally observed PL decay. In the inset in Fig. 2(a), we compare the normalized FEC signals at 5 and $10 \mathrm{~mW}$. The lack of excitation-intensity dependence of the FEC-signal shapes implies that the excitonexciton annihilation or Auger recombination process does not appear in the exciton dynamics within this excitationintensity region $[33,34]$.
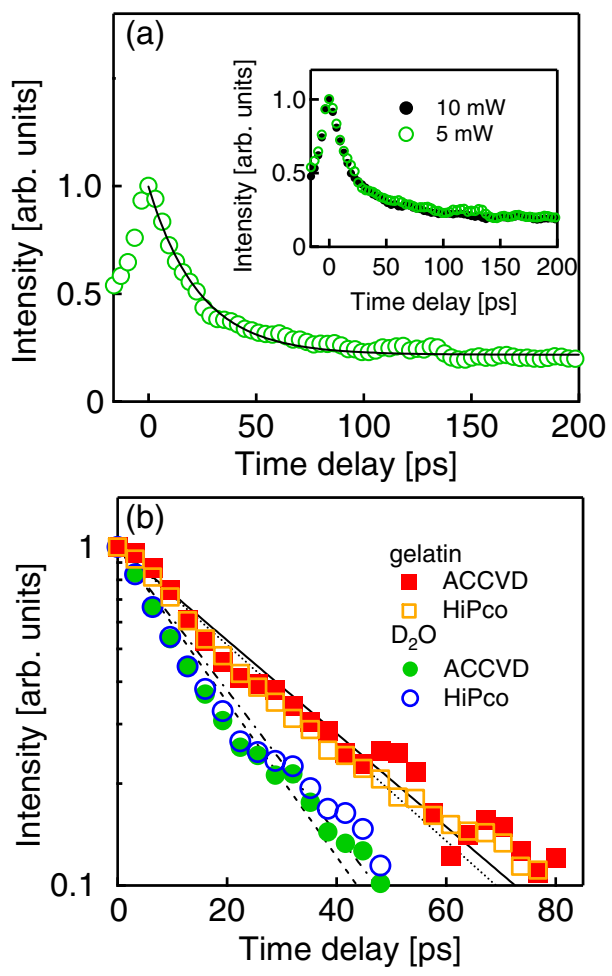

FIG. 2 (color online). (a) Normalized FEC signals for $(9,4)$ nanotubes of ACCVD-D $\mathrm{D}_{2} \mathrm{O}$ samples measured under $1.70 \mathrm{eV}$ excitation at $5 \mathrm{~mW}$. The solid line is given by the function of $A \exp \left(-\tau / \tau_{\mathrm{PL}}\right)+B$. The inset shows the normalized FEC signals measured at different excitation intensities $(5$ and $10 \mathrm{~mW}$ at $1.70 \mathrm{eV})$. (b) Normalized fast-decay components of the FEC signals from $(9,4)$ nanotubes of the ACCVD-gelatin, HiPcogelatin, ACCVD- $\mathrm{D}_{2} \mathrm{O}$, and $\mathrm{HiPco}-\mathrm{D}_{2} \mathrm{O}$ samples measured with an optical pulse at $1.70 \mathrm{eV}$. The solid (ACCVD-gelatin), dotted (HiPco-gelatin), dashed (ACCVD- $\left.\mathrm{D}_{2} \mathrm{O}\right)$, and dotted-dashed (HiPco- $\mathrm{D}_{2} \mathrm{O}$ ) lines represent the normalized fast-decay components of fitting curves, $A \exp \left(-\tau / \tau_{\mathrm{PL}}\right)$, respectively. The excitation optical pulse intensity is $5 \mathrm{~mW}$. The upward direction on the vertical axis indicates a negative sign of the FEC signals. 
Figure 2(b) shows the fast-decay components of the FEC signals as a function of delay time for four kinds of samples with $(9,4)$ nanotubes. PL lifetimes $\tau_{\mathrm{PL}}$ are derived as 32 (ACCVD-gelatin), 30 (HiPco-gelatin), 19 (ACCVD- ${ }_{2} \mathrm{O}$ ), and 21 ps $\left(H i P c o-\mathrm{D}_{2} \mathrm{O}\right)$ [24]. The PL lifetimes show that the fast-decay components scarcely depend on the synthesis method of SWNTs but depend more on the environment. Our observation shows that the number of defects and impurities related to the nonradiative centers in the ACCVD-SWNTs is almost the same as those in HiPcoSWNTs and that the slower PL decay in the solid films is determined by local potential fluctuations due to strains, dielectric constants, and structural defects. The PL lifetimes $\tau_{\mathrm{PL}}$ of the fast-decay components are similar to the nonradiative decay lifetimes $\tau_{\mathrm{PL}}(10-180 \mathrm{ps})$ measured in previous experiments [14-19].

Figure 3 shows the PL lifetimes of the fast-decay components as a function of the tube diameter. In Fig. 3, no significant dependence of $\tau_{\mathrm{PL}}$ on tube diameter is observed within experimental errors. The results, which are consistent with those of other experiments [16], imply that the recombination rate is not sensitive to the tube diameter. As shown in Fig. 3, the mean PL lifetime of the SWNTs in $\mathrm{D}_{2} \mathrm{O}$ solutions $\left(\tau_{\mathrm{PL}} \sim 22 \mathrm{ps}\right)$ is faster than that in gelatin films $\left(\tau_{\mathrm{PL}} \sim 31 \mathrm{ps}\right)$. This difference supports the idea that the PL lifetime is sensitive to the environment.

In addition, we studied the temperature dependence of the fast-decay components of FEC signals to elucidate the detailed mechanism of the exciton dynamics. Figure 4(a) shows the fast-decay components of FEC signals of $(9,4)$ nanotubes in the ACCVD-gelatin samples at different temperatures [35]. With an increase in temperature, the PL lifetime $\tau_{\mathrm{PL}}$ becomes shorter. Figure 4(b) shows the relaxation rate $1 / \tau_{\mathrm{PL}}$ as a function of temperature. As shown in Figs. 4(a) and 4(b), the relaxation rate $1 / \tau_{\mathrm{PL}}$ gradually decreases with a decrease in temperature. This temperature dependence cannot be explained by the radiative decay rate

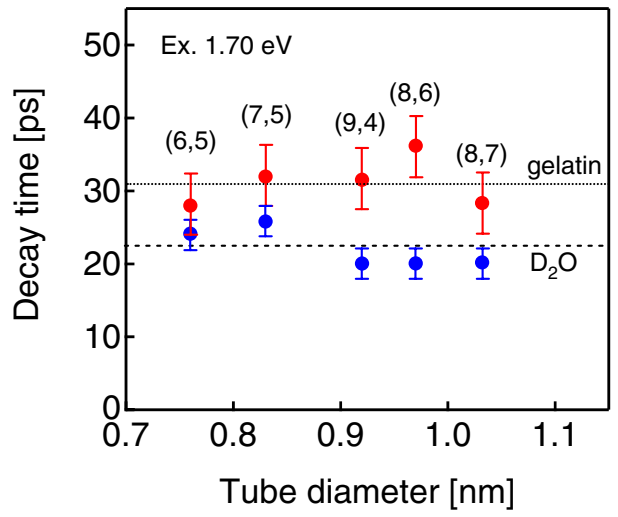

FIG. 3 (color online). PL lifetimes $\tau_{\mathrm{PL}}$ of the fast-decay components of the FEC signals as a function of tube diameter in the ACCVD-gelatin and ACCVD- $\mathrm{D}_{2} \mathrm{O}$ samples. The dotted (ACCVD-gelatin) and dashed (ACCVD- $\mathrm{D}_{2} \mathrm{O}$ ) lines represent the mean values of PL lifetimes for five nanotube species with different diameters.
$1 / \tau_{r}$ of the excitons in the 1D systems, because it increases with decreasing temperature and is inversely proportional to the square root of the temperature [36]. Instead, our results imply the importance of the nonradiative recombination process in the fast-decay component. In addition, the PL spectrum and dynamics depend on the environments of the SWNTs, as shown in Figs. 1 and 2. Furthermore, the weak temperature dependence of PL lifetimes, shown in Fig. 4(b), is suggestive of weakly localized excitons due to environmental fluctuations in the wrapping gelatin.

Hence, the PL dynamics in SWNTs can be explained by the interplay between localized excitons and free excitons. The inset in Fig. 4(b) contains a schematic diagram illustrating the dynamics of localized and free excitons in SWNTs. Excited excitons, captured by localized exciton states $(L)$, are thermally excited into free exciton states $(F)$. In this case, the observed PL lifetime $\tau_{\mathrm{PL}}$ can be approximated by Eq. (1):

$$
\tau_{\mathrm{PL}}^{-1} \approx \tau_{L}^{-1}+\tau_{F}^{-1} \exp \left(-\Delta / k_{B} T\right),
$$
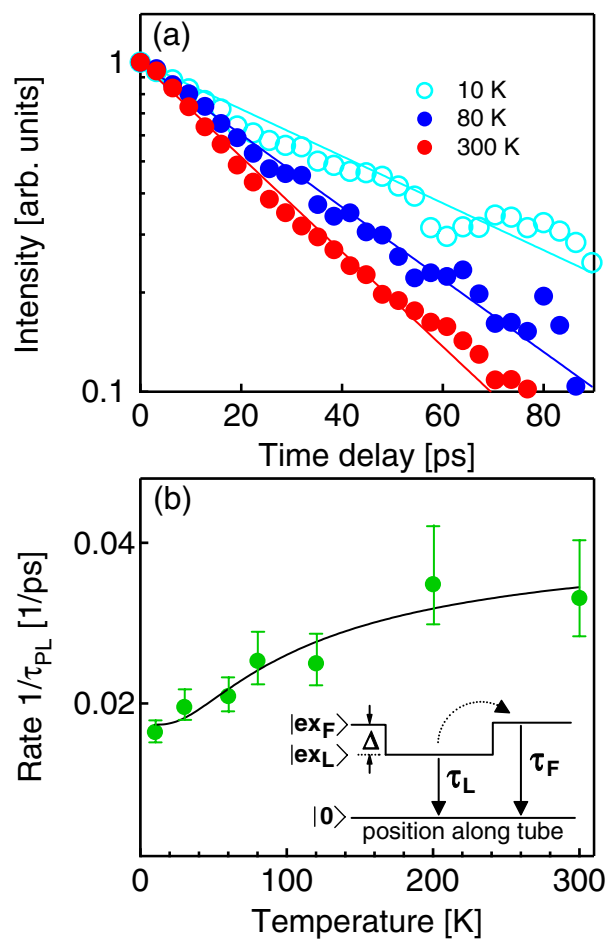

FIG. 4 (color online). (a) Normalized fast-decay components of the FEC signals from $(9,4)$ nanotubes of the ACCVD-gelatin samples at 10,80 , and $300 \mathrm{~K}$. The solid lines represent the normalized fast-decay components of fitting curves, $A \exp \left(-\tau / \tau_{\mathrm{PL}}\right)$, respectively. The excitation intensity is $1 \mathrm{~mW}$ at $1.70 \mathrm{eV}$. The upward direction on the vertical axis indicates a negative sign of the FEC signals. (b) The temperature dependence of the relaxation rate $1 / \tau_{\mathrm{PL}}$ between 10 and $300 \mathrm{~K}$ for $(9,4)$ nanotubes of the ACCVD-gelatin samples. The solid line shows the relaxation rate described by Eq. (1). The inset contains a schematic sketch illustrating the dynamics of localized exciton $\left|e x_{L}\right\rangle$ and free excitons $\left|e x_{F}\right\rangle$ in SWNTs. 
where $k_{B}$ is the Boltzmann constant, $\Delta$ is thermal activation energy, and $\tau_{\mathrm{L}}$ and $\tau_{F}$ are the PL lifetimes determined mainly by the nonradiative recombination processes of localized excitons and free excitons, respectively. As shown in Fig. 4(b), Eq. (1) reproduces the experimental data, where the best fitting parameters are $\tau_{L}=58 \mathrm{ps,}$ $\tau_{F}=42 \mathrm{ps}$, and $\Delta=8.7 \mathrm{meV}$. The uncertainties are $\sim \pm$ $10 \%$, owing mainly to errors in the measurement of $\tau_{\mathrm{PL}}$ due to the macroscopic inhomogenity in gelatin samples. At present, it is believed that the energy difference $\Delta$ between the low-energy and the high-energy exciton states reflects the sample-dependent extrinsic effects (the formation of the localized states) rather than the intrinsic effects such as the exchange splitting of the dark and bright excitons [20,21], because the PL dynamics depends on the local environment surrounding the SWNTs as discussed above. The small activation energy $\Delta$ suggests that the excited excitons are weakly localized by local environmental fluctuations, mostly due to the local changes in strains, dielectric constants, and structural defects along a nanotube. Studying the PL decays of SWNTs in other matrices and surfactants may be helpful to obtain a more quantitative and microscopic nature on the localized states.

Moreover, as shown in Fig. 1, it is evident that the PL spectrum of SWNTs in $\mathrm{D}_{2} \mathrm{O}$ solutions has a sharp structure and shows small blueshifts in comparison with that in gelatin films. This observation suggests that the activation energy $\Delta$ of the potential fluctuations in SWNTs in $\mathrm{D}_{2} \mathrm{O}$ solutions becomes relatively small. In such a case, at room temperature the recombination process of excitons is promoted, because the decay rate of free excitons is larger than that of localized excitons. Therefore, we can observe the difference between the PL lifetime of SWNTs in $\mathrm{D}_{2} \mathrm{O}$ and that of SWNTs in gelatin in Figs. 2 and 3, and this difference is mainly due to the difference of their activation energy $\Delta$ depending on the surrounding matrices.

In conclusion, we measured the PL lifetimes in the SWNTs by the femtosecond excitation correlation method with a 150 fs time resolution. The SWNTs were synthesized by different methods (ACCVD or HiPco) and suspended in gelatin films or $\mathrm{D}_{2} \mathrm{O}$ solutions. The typical PL lifetime of the SWNTs in $\mathrm{D}_{2} \mathrm{O}$ solutions $\left(\tau_{\mathrm{PL}} \sim 22 \mathrm{ps}\right)$ is faster than that in gelatin films $\left(\tau_{\mathrm{PL}} \sim 31 \mathrm{ps}\right)$ but scarcely depends on the synthesis method and the tube diameter of SWNTs. The very weak temperature dependence of $\tau_{\mathrm{PL}}$ and the environment-dependent $\tau_{\mathrm{PL}}$ reveal that the recombination dynamics of excitons in SWNTs is determined by the competing process between free excitons and weakly localized excitons rather than the intrinsic effects such as the exchange splitting of excitons.

Part of this work at Kyoto University is supported by MEXT Joint Project of Chemical Synthesis Core Research Institutions, the Grant-in-Aid for Scientific Research from JSPS (No. 17684012 and No. 18340089), the Foundation for C\&C Promotion, and the Research Foundation for Opto-Science and Technology.
*Electronic address: matsuda@scl.kyoto-u.ac.jp

${ }^{\dagger}$ Electronic address: kanemitu@scl.kyoto-u.ac.jp

[1] S. Iijima, Nature (London) 354, 56 (1991).

[2] R. Saito, G. Dresselhaus, and M. S. Dresselhaus, Physical Properties of Carbon Nanotubes (Imperial College Press, London, 1998).

[3] M. J. O'Connell et al., Science 297, 593 (2002).

[4] T. Ando, J. Phys. Soc. Jpn. 66, 1066 (1997).

[5] T. Ogawa, in Optical Properties of Low-Dimensional Materials, edited by T. Ogawa and Y. Kanemitsu (World Scientific, Singapore, 1995), p. 1.

[6] T. G. Pedersen, Phys. Rev. B 67, 073401 (2003).

[7] C. L. Kane and E. J. Mele, Phys. Rev. Lett. 93, 197402 (2004).

[8] C. D. Spataru et al., Phys. Rev. Lett. 92, 077402 (2004).

[9] V. Perebeinos, J. Tersoff, and P. Avouris, Phys. Rev. Lett. 92, 257402 (2004).

[10] H. Zhao and S. Mazumdar, Phys. Rev. Lett. 93, 157402 (2004).

[11] E. B. Barros et al., Phys. Rev. B 73, 241406(R) (2006).

[12] F. Wang et al., Science 308, 838 (2005).

[13] J. Maultzsch et al., Phys. Rev. B 72, 241402(R) (2005).

[14] F. Wang et al., Phys. Rev. Lett. 92, 177401 (2004).

[15] A. Hagen et al., Appl. Phys. A 78, 1137 (2004).

[16] S. Reich et al., Phys. Rev. B 71, 033402 (2005).

[17] T. Hertel et al., Nano Lett. 5, 511 (2005).

[18] M. Jones et al., Phys. Rev. B 71, 115426 (2005).

[19] A. Hagen et al., Phys. Rev. Lett. 95, 197401 (2005).

[20] C. D. Spataru et al., Phys. Rev. Lett. 95, 247402 (2005).

[21] V. Perebeinos, J. Tersoff, and P. Avouris, Nano Lett. 5, 2495 (2005).

[22] Y. Murakami et al., Chem. Phys. Lett. 374, 53 (2003).

[23] W. Zhou et al., Chem. Phys. Lett. 350, 6 (2001).

[24] We confirmed that the PL lifetime of the SDS-suspended SWNT solutions is almost the same as that of the SDBSsuspended SWNT solutions.

[25] M. Jørgensen and J. M. Hvam, Appl. Phys. Lett. 43, 460 (1983).

[26] The PL intensity of suspended SWNT solution gradually decreases in the initial stage just after preparing them and reaches a constant value as time passes; hence, all experiments are conducted using the samples showing stable PL.

[27] S. M. Bachilo et al., Science 298, 2361 (2002).

[28] R. B. Weisman and S.M. Bachilo, Nano Lett. 3, 1235 (2003).

[29] Y. Miyauchi et al., Chem. Phys. Lett. 387, 198 (2004).

[30] V.C. Moore et al., Nano Lett. 3, 1379 (2003).

[31] L.-J. Li et al., Phys. Rev. Lett. 93, 156104 (2004).

[32] O. J. Korovyanko et al., Phys. Rev. Lett. 92, 017403 (2004).

[33] L. Valkunas, Y.-Z. Ma, and G. R. Fleming, Phys. Rev. B 73, 115432 (2006).

[34] F. Wang et al., Phys. Rev. B 70, 241403(R) (2004).

[35] We measured the signals at a fixed energy from 10 to $300 \mathrm{~K}$ for each tube, because the peak energy hardly changes within our experimental temperature range. We did not observe the large energy shift [see D. Karaiskaj et al., Phys. Rev. Lett. 96, 106805 (2006)] in our SWNT in gelatin.

[36] D. S. Citrin, Phys. Rev. Lett. 69, 3393 (1992). 